\title{
Hepatic ultrastructure on Wilson's disease (WD) suspicious patients.
}

Sara Hilda Mandado Pérez*, Antonio Pérez Brioso*, Carlos Domínguez Alvarez**, Bienvenido Grá Oramas*, Felicia Pomar Montalvo* y Clara Melendi Delgado**.

Instituto Nacional de Gastroenterología*. Hospital Clínico Quirúrgico Hermanos Ameijeiras**.

Introduction: WD is an autonomic recessive disorder caused by mutations in the ATP7Bgene, characterized by dramatic of hepatic accummulations of hepatic copper, with subsequent hepatic and neurologic abnormalities. Electron mycroscopy has been decisive for Wilson's disease precocious diagnosis. Three stages have been morphologically identified depending on mitochondrial and lisosomal damage.

Wilson's disease early diagnosis is very important because it enables the application of an adequate therapy and avoids fatal outcomes.

Objective: To study the fine structure of WD hepatic tissue in order to improve the morfologic criteria for early diagnosis.

Materials and methods: Samples of hepatic biopsies from 18 adult patients (males and females) clinically suspicious of WD were studied. Biopsies were obtained by laparoscopy and divided into three fragments: for optical and electron microscopy and for tissue determination of copper.

Results: In all cases the liver copper content was increased and WD was confirmed clinically.

At optical microscope 10 out of the 18 cases showed morphological alterations compatible with WD; 6 of them showed ultrastructural features typical of WD. The other 8 ( of the 18) were diagnosed as normal under the optical microscope, but 6 of them presented outstanding mitochondrial alterations, among them the three typical kinds of intramitochondrial inclusions. The latter was determinant for the early diagnosis of WD.

Conclusions: Electron microscopy is highly valuable for Wilson's disease early diagnosis, especially for those patients whose biopsies do not show histologic alterations visible at the optical microscope. 


\section{( En Español)}

\section{ULTRAESTRUCTURA DEL TEJIDO HEPATICO EN PACIENTES SOSPECHOSOS DE ENFERMEDAD DE WILSON.}

Autores: Sara Hilda Mandado Pérez*, Antonio Pérez Brioso**, Carlos Domínguez Alvarez***, Bienvenido Grá Oramas****, Felicia Pomar Montalvo***** y Clara Melendi Delgado $* * * * * *$.

Instituciones : Instituto Nacional de Gastroenterología. Hospital Clínico Quirúrgico Hermanos Ameijeiras.

\section{Resumen}

Introduction: La Enfermedad de Wilson (EW) es una anormalidad hereditaria, autosómica recesiva, cuyo gen responsable codifica para la ATPasa tipoP transportadora de cobre (ATPasa 7B) en el cromosoma 13q14.3, que se manifiesta en alteraciones del metabolismo del cobre; cuyas dos características principales son los niveles bajos de ceruloplasmina en suero y el depósito excesivo de cobre $(\mathrm{Cu})$ en los tejidos, sobre todo en el parénquima hepático. La excreción urinaria de cobre es alta, la biliar baja y los niveles séricos bajos. La microscopía electrónica ha contribuido al diagnóstico precoz de la enfermedad, y se ha establecido la caractaerización estructural de sus tres estadios, basada fundamentalmente en las alteraciones mitocondriales y lisosomicas de los hepatocitos. El diagnóstico en el estadio inicial de la EW es primordial para aplicar oportunamente el tratamiento con quelantes del cobre y evitar un desenlace fatal.

Objetivos : Estudiar la estructura fina del tejido hepático en pacientes sospechosos de EW para contrbuir al diagnóstico precoz de la enfermedad.

Materiales y métodos: se estudió la estructura fina del tejido hepático de 18 pacientes adultos,de ambos sexos, clínicamente sospechosos de EW. La biopsia fue tomada mediante laparoscopia y dividida dividida en tres fragmentos para estudio ultraestructural, histológico y dosificación de cobre en tejido. Las muestra para microscopía electrónica fueron fijadas en Glutaraldehido- Osmio e incluidas en araldita.

Resultados: En los 18 casos las cifras de cobre en tejido fueron elevadas y el diagnóstico de EW fue establecida clínicamente.

Al microscopio óptico 10 de los 18 casos presentaron caracteres morfológicos compatibles con EW; 6 de ellos mostraron alteraciones ultraestructurales típicas de EW. Los 8 casos restantes no presentaban lesiones histológicas visbles al microscopio óptico, pero mostraban las alteraciones mitocondriales más llamativas, entre ellas los tres tipos de inclusiones intramitocondriales, que fueron determinantes para el diagnóstico precoz de la enfermedad

Conclusiones: La microscopía electrónica es de gran utilidad en el diagnóstico precoz de la $\mathrm{EW}$, especialmente en los pacientes que no presentan lesiones visibles al microscopio óptico.

*Licenciada en Biología. Investigadora Agregada. IGE.**Especialista de $2^{\circ}$ Grado en Gastroenterología.. IGE.***Especialista $2^{\circ}$ Grado en Patología. Investigador Auxiliar. Prof. Auxiliar. J' Sección de Microscopía Electrónica.HCQ Hnos Ameijeiras.**** Especialista $2^{\circ}$ Grado en Patología. Investigador Auxiliar. Prof. Auxiliar. Subd. de Investigaciones. IGE*****Lic. en Bioquímica. Investigadora Agregada. IGE.******Lic. en Tecnología de la Salud. HCQ Hnos Ameijeiras. 